\title{
TESTES DE VIGOR PARA SEMENTES DE CENOURA
}

\section{VIGOR TESTS FOR CARROT SEEDS}

\author{
Cristina Batista de LIMA ${ }^{1}$ \\ João Carlos ATHANÁZIO²
}

\section{RESUMO}

O trabalho teve por objetivo comparar testes de vigor para a análise da qualidade fisiológica de sementes de cenoura, classificadas por tamanho e armazenadas por diferentes períodos. Seis lotes de sementes população 'Londrina' foram submetidos a testes de germinação, tetrazólio, envelhecimento acelerado com água, com solução salina e emergência de plântulas. O teste de emergência de plântulas destaca-se na classificação dos lotes quanto às diferenças de vigor. $\mathrm{A}$ combinação solução salina com 48 ou $72 \mathrm{~h}$ de exposição é a mais adequada para o teste de envelhecimento acelerado. As sementes de maior tamanho apresentam qualidade fisiológica superior às menores.

Palavras-chave: Qualidade fisiológica; germinação; Daucus carota L.

\begin{abstract}
This work had the purpose to compare the efficiency of vigor tests for evaluation of physiological quality of carrot seeds, ranked for size and stored by different periods. Six lots of carrot seeds from 'Londrina' population were tested for germination, tetrazolium, accelerated aging traditional, accelerated aging modified and seedling emergency. The seedling emergence test is distinguished in classification the lots by the vigor differences. The combination salt solution with 48 or $72 \mathrm{~h}$ of exposure is adjusted for the accelerated aging test. The seeds of larger size have higher physiological quality to smaller.
\end{abstract}

Key-words: Physiological quality; germination; Daucus carota L.

\footnotetext{
${ }^{1}$ Departamento de Produção Vegetal, Universidade Estadual do Norte do Paraná- Campus “Luiz Meneghel”, BR-369, km 54, C.P. 261, CEP: 86360-000, Bandeirantes, Paraná, Brasil. E-mail: crislima@ffalm.br. Autor para correspondência
}

${ }^{2}$ Centro de Ciências Agrárias da Universidade Estadual de Londrina (UEL), CEP:86051-990, Londrina, Paraná, Brasil.E-mail: jcatha@uel.br

Scientia Agraria, Curitiba, v.10, n.6, p.455-461, Nov./Dec. 2009. 
LIMA, C.B. \& ATHANÁZIO, J.C. Testes de vigor para sementes...

\section{INTRODUÇÃO}

As plantas de cenoura apresentam amplo período de florescimento gerando elevada desuniformidade de vigor nas sementes, ainda que sejam produzidas sob mesmas condições edafoclimáticas e derivadas de mesma população. Esse fato tem proporcionado dificuldades para o cultivo dessa planta, contribuindo para a obtenção de baixos estandes ou gasto exagerado de sementes encarecendo o custo de produção (Vieira et al., 2005). Neste sentido, é necessário o desenvolvimento de procedimentos para avaliar a qualidade dessas sementes.

A qualidade de sementes é a soma dos atributos que contribuem para o seu desempenho do armazenamento à germinação, sendo particularmente importante para sementes de hortaliças, cuja demanda é estável ao longo do ano, enquanto a produção varia acentuadamente a cada safra (Ramos et al., 2004). O teste de germinação, normalmente utilizado na avaliação da qualidade de sementes, permite que o lote expresse sua máxima porcentagem de germinação. Entretanto, em situações de campo, as circunstâncias nem sempre favoráveis, fazem com que ocorram discrepâncias em relação aos resultados obtidos em laboratório (Hilhorst et al., 2001).

Assim, os testes de vigor foram desenvolvidos com 0 objetivo de identificar possíveis diferenças no grau de deterioração de sementes que apresentem potencial germinativo semelhante, podendo estimar sua capacidade de armazenamento e emergência de plântulas em campo. Alguns desses testes têm sido indicados para sementes de hortaliças, porém, não podem ser generalizados, pois os resultados de pesquisas disponíveis são insuficientes para a definição de metodologia apropriada (Franzin et al., 2004).

Dentre os métodos propostos para estimar o vigor de sementes da maioria das olerícolas e demais espécies com sementes pequenas, o envelhecimento acelerado apresenta limitações, em função da rápida absorção de água por essas sementes durante o teste. Esse fato intensifica a deterioração, resultando em percentuais muito baixos de germinação pós-envelhecimento (Muniz et al., 2004). Para controlar este fator, tem-se utilizado soluções saturadas de sais durante a realização do teste (Jianhua \& McDonald, 1996); procedimento este considerado eficiente na classificação do vigor de lotes de sementes de cenoura (Rodo et al., 2000).

O tamanho da semente é considerado um indicativo de sua qualidade fisiológica sendo que as maiores, normalmente, possuem embriões bem formados com elevados potenciais de vigor e germinação, logo, num mesmo lote, as sementes maiores, potencialmente, apresentam maior poder germinativo e vigor que as sementes menores, embora existam controvérsias quanto aos benefícios da classificação de sementes de cenoura por tamanho (Rodo et al., 2001).

Considerando que, germinação e vigor de sementes têm reflexos no desempenho da plântula; assim há a necessidade de estabelecer métodos eficientes para avaliação dessas características. $O$ presente trabalho teve por objetivo comparar diferentes métodos para avaliação do vigor de sementes de cenoura, separadas por tamanho e armazenadas por diferentes períodos.

\section{MATERIAL E MÉTODOS}

O trabalho foi conduzido no Laboratório de Análise de Sementes do Departamento de Produção Vegetal, da Universidade Estadual do Norte do Paraná - Campus Luiz Meneghel. Foram utilizados seis lotes de uma população regional de sementes de cenoura denominada 'Londrina' produzidas no sistema semente-raiz-semente, nas safras correspondentes aos anos de 1986 a 2004, no campus da Universidade Estadual de Londrina UEL. Após a colheita, as sementes foram beneficiadas, acondicionadas em sacos de papel Kraft e mantidas sob temperatura de $10^{\circ} \mathrm{C}$.

Inicialmente amostras de sementes das safras de 1986 a 2004 foram submetidas à análise de germinação, para seleção das sementes viáveis. Dessa análise foram selecionadas sementes das safras de 1999 a 2004, por apresentarem os maiores percentuais de germinação, obtendo-se assim, os seis lotes de sementes utilizados nesta pesquisa. As sementes dos lotes selecionados foram submetidas as seguintes determinações:

- Separação por tamanho: as sementes de cada lote foram separadas com peneiras manuais nas frações grande (retidas em peneira de perfuração redonda $1,41 \mathrm{~mm}$ - ABNT 14) e pequena (retidas em peneira de perfuração redonda $1,00 \mathrm{~mm}$ - ABNT 18).

- Massa de mil sementes: foram avaliadas oito repetições de 100 sementes e o resultado foi obtido multiplicando-se por 10 a massa média de oito subamostras de 100 sementes de cada lote, conforme BRASIL (1992).

- Teor de água: método da estufa a $105 \pm 3$ ${ }^{\circ} \mathrm{C}$, durante $24 \mathrm{~h}$, com duas subamostras de $5 \mathrm{~g}$ para cada lote (BRASIL, 1992). Esse procedimento foi realizado antes e após os testes de envelhecimento acelerado com água e solução salina. Os resultados foram expressos em porcentagem na base úmida.

Germinação: conduzido com quatro repetições de 50 sementes, distribuídas em caixas plásticas, sobre papel filtro umedecido com água destilada na proporção de 2,5 vezes a sua massa seca, mantidas sob temperatura constante de 20 ${ }^{\circ} \mathrm{C}$. As contagens foram realizadas no sétimo e décimo quarto dias após a instalação e as avaliações efetuadas segundo os critérios estabelecidos pelas RAS para essa espécie (BRASIL, 1992).

- Tetrazólio: realizado com duas repetições de 50 sementes cada, as quais foram previamente colocadas em água destilada, durante $18 \mathrm{~h}$ a $25^{\circ} \mathrm{C}$. Após esse período, as sementes foram seccionadas por um corte longitudinal e lateral ao embrião e, imediatamente, embebidas na solução de tetrazólio 
a $1 \%$, durante $24 \mathrm{~h}$ a $30^{\circ} \mathrm{C}$. A seguir a solução de tetrazólio foi drenada e as sementes lavadas em água destilada e seccionadas por um corte longitudinal, através do embrião para análise de viabilidade (BRASIL, 1992). O percentual de sementes viáveis foi determinado, através de um estereomicroscópio, com ampliação entre 20 e 100 vezes o tamanho original. A determinação das categorias foi estabelecida de acordo com Andrade et al. (1996).

- Primeira contagem: realizada juntamente com o teste de germinação, pelo registro do número de plântulas normais presentes no sétimo dia após o início do teste.

- Velocidade da germinação: o número de plântulas normais foi registrado diariamente, no mesmo horário, do $1^{\circ}$ ao $14^{\circ}$ dia do início do teste de germinação, para o estabelecimento do índice de velocidade de germinação, calculado de acordo com Popinigis (1985).

- Envelhecimento acelerado: conforme metodologia descrita por Marcos Filho (1999) foram distribuídas 200 sementes sobre tela metálica, acoplada a caixas plásticas, contendo $40 \mathrm{~cm}^{3}$ de água destilada no fundo. Os recipientes foram mantidos sob temperatura de $42{ }^{\circ} \mathrm{C}$ em câmara de germinação, durante $12,24,36,48,60,72$ e 84 h. Ao término de cada período, as sementes foram avaliadas pelo teste de germinação. No envelhecimento acelerado com solução salina, o procedimento anterior foi repetido, substituindo-se os $40 \mathrm{~cm}^{3}$ de água destilada adicionados às caixas plásticas por $40 \mathrm{~cm}^{3}$ de solução saturada de $\mathrm{NaCl}$ (40 $\mathrm{g}$ do sal $100 \mathrm{~cm}^{-3}$ de água), conforme Jianhua \& McDonald (1996).
- Emergência de plântulas: conduzido com duas amostras de 50 sementes de cada lote, semeadas em bandejas de isopor de 200 células, preenchidas com um substrato composto por solo, areia e matéria orgânica na proporção de 1:1:1, a $0,5 \mathrm{~cm}$ de profundidade. As bandejas foram mantidas em casa de vegetação e irrigadas diariamente. O número de plântulas normais emergidas foi contado diariamente no mesmo horário, do $1^{\circ}$ ao $14^{\circ}$ dia após a semeadura, para estabelecimento do índice de velocidade de emergência, calculado segundo Popinigis (1985).

O delineamento experimental foi 0 inteiramente casualizado, os dados obtidos em cada teste foram submetidos à análise de variância e as médias comparadas pelo teste de Tukey, a 5\% de probabilidade. O grau de associação entre os resultados foi analisado pela técnica de correlação simples de Pearson ( $r$ ). Os dados referentes ao grau de umidade não foram analisados, servindo para caracterização inicial dos lotes e monitoramento, durante o teste de envelhecimento acelerado.

\section{RESULTADOS E DISCUSSÃO}

Os lotes com 24, 48 e 60 meses de armazenamento apresentaram um percentual médio de germinação acima do padrão mínimo de $65 \%$, estabelecido para a comercialização de sementes de cenoura (Tabela 1). A tendência de que sementes novas têm maior vigor não foi constatada, pois, as sementes com 60 e 48 meses de armazenamento, demonstraram percentual de germinação superior às com 24 meses.

TABELA 1 - Características descritivas físicas e fisiológicas de seis lotes de sementes de cenoura população 'Londrina'.

\begin{tabular}{lcccccc}
\hline \multirow{2}{*}{ Características } & \multicolumn{9}{c}{ Lotes } \\
\cline { 2 - 7 } & 1 & 2 & 3 & 4 & 5 & 6 \\
\hline Armazenamento (meses) & 84 & 72 & 60 & 48 & 36 & 24 \\
Teor de água (\%) & 9,5 & 8,7 & 8,9 & 9,7 & 8,9 & 8,9 \\
Germinação (\%) & 2 & 25 & 84 & 80 & 60 & 76 \\
Massa 1000 sementes grandes $(\mathrm{g})^{1}$ & 1,89 & 1,97 & 2,41 & 2,07 & 1,97 & 2,46 \\
Massa 1000 sementes pequenas $(\mathrm{g})^{2}$ & 1,45 & 1,38 & 1,69 & 1,80 & 1,39 & 1,54 \\
\hline
\end{tabular}

${ }^{1}$ Sementes retidas em peneira de perfuração redonda 1,41 $\mathrm{mm}(\mathrm{ABNT} 14)$

2 Sementes retidas em peneira de perfuração redonda $1,00 \mathrm{~mm}(\mathrm{ABNT} 18)$

Pelos resultados do teste de tetrazólio (Tabela 2), as principais causas da baixa viabilidade foram 0 alto percentual de sementes mortas e a falta de material de reserva. A ocorrência de sementes sem embrião para ambos os tamanhos, apresentou uma amplitude de zero a onze. Andrade et al. (1996), descrevem esta anormalidade como sendo de alta herdabilidade, com origem e causa pouco esclarecidas. Os resultados descritos no teste de tetrazólio permitem a separação dos lotes em dois grupos em função de sua viabilidade.

Os testes de germinação e primeira contagem da germinação classificaram os lotes de modo diferenciado, apresentando três classes para sementes grandes e quatro para as sementes pequenas. Rodo et al. (2001), não obtiveram resultados satisfatórios quanto à separação dos lotes de sementes de cenoura, através do teste de germinação. Os resultados do teste de germinação mesmo indicando diferenças estatísticas entre lotes, não garantem desempenho posterior similar, pois o comportamento das sementes depende das condições ambientais presentes na semeadura e no desenvolvimento das plântulas em campo. 
LIMA, C.B. \& ATHANÁZIO, J.C. Testes de vigor para sementes...

TABELA 2 - Viabilidade e caracterização fisiológica (médias percentuais) de sementes grandes e pequenas de cenoura, através dos testes de tetrazólio ( $V=$ viáveis; $M=$ mortas; $S E=s e m$ endosperma; $E M=e m b r i a ̃ o$ morto; $\mathrm{SB}=$ sem embrião), germinação $(\mathrm{G})$, primeira contagem da germinação (PCG); índice de velocidade de germinação (IVG); emergência de plântulas (EP) e índice de velocidade de emergência (IVE) de seis lotes de sementes de cenoura população 'Londrina'.

\begin{tabular}{|c|c|c|c|c|c|c|c|c|c|c|}
\hline \multirow{3}{*}{ Lotes } & \multicolumn{5}{|c|}{ Tetrazólio } & \multicolumn{3}{|c|}{ Germinação } & \multicolumn{2}{|c|}{ Emergência } \\
\hline & v & M & SE & EM & SB & G & PCG & IVG & EP & IVE \\
\hline & \multicolumn{10}{|c|}{ Sementes grandes } \\
\hline 1 & $7,0 b^{1}$ & $37,0 \mathrm{~b}$ & $44,0 \mathrm{a}$ & $12,0 \mathrm{a}$ & $0,0 \mathrm{~b}$ & $0 \mathrm{c}$ & $0 \mathrm{c}$ & $0,0 \mathrm{c}$ & $2 f$ & $0,2 f$ \\
\hline 2 & $10,0 \mathrm{~b}$ & $62,0 \mathrm{a}$ & $16,0 \mathrm{~b}$ & $1,0 \mathrm{~b}$ & $11 \mathrm{a}$ & $9 c$ & $5 c$ & $1,4 \mathrm{c}$ & 33 e & $3,9 \mathrm{e}$ \\
\hline 3 & $66,0 \mathrm{a}$ & $24,0 \mathrm{bc}$ & $9,0 \mathrm{~b}$ & $1,0 \mathrm{~b}$ & $0,0 \mathrm{~b}$ & $71 \mathrm{a}$ & $69 \mathrm{a}$ & $20,2 \mathrm{a}$ & 99 a & $14,7 \mathrm{a}$ \\
\hline 4 & $24,0 \mathrm{~b}$ & $58,0 \mathrm{a}$ & $14,0 \mathrm{~b}$ & $2,0 \mathrm{~b}$ & $2,0 \mathrm{~b}$ & $42 \mathrm{~b}$ & $40 \mathrm{~b}$ & $12,2 \mathrm{~b}$ & $76 d$ & $12,1 \mathrm{~d}$ \\
\hline 5 & $71,0 \mathrm{a}$ & $18,0 \mathrm{c}$ & $9,0 \mathrm{~b}$ & $0,0 \mathrm{~b}$ & $2,0 \mathrm{~b}$ & $57 \mathrm{ab}$ & $54 \mathrm{ab}$ & $13,3 b$ & $95 \mathrm{~b}$ & $13,9 b$ \\
\hline 6 & $55,0 \mathrm{a}$ & $13,0 \mathrm{c}$ & $25,0 \mathrm{ab}$ & $2,0 \mathrm{~b}$ & $5,0 \mathrm{~b}$ & $50 \mathrm{~b}$ & $47 \mathrm{~b}$ & $13,2 b$ & $89 c$ & $12,4 \mathrm{c}$ \\
\hline CV\% & 18,9 & 11,6 & 26,9 & 60,8 & 42,4 & 16,1 & 17,9 & 19,1 & 30,4 & 18,2 \\
\hline \multicolumn{11}{|c|}{ Sementes pequenas } \\
\hline 1 & $25,0 \mathrm{~b}$ & $40,0 \mathrm{ab}$ & $23,0 \mathrm{ab}$ & $4,0 \mathrm{~b}$ & $8,0 \mathrm{a}$ & $4 d$ & $2 d$ & $0,5 \mathrm{c}$ & $3 f$ & $0,3 \mathrm{f}$ \\
\hline 2 & $30,0 \mathrm{~b}$ & $55,0 \mathrm{a}$ & $6,0 \mathrm{~b}$ & $1,0 \mathrm{~b}$ & $8,0 \mathrm{a}$ & $10 d$ & $7 d$ & $1,5 \mathrm{c}$ & $29 \mathrm{e}$ & $3,0 \mathrm{e}$ \\
\hline 3 & $73,0 \mathrm{a}$ & $18,0 \mathrm{bc}$ & $8,0 \mathrm{~b}$ & $0,0 \mathrm{~b}$ & $1,0 \mathrm{a}$ & $66 \mathrm{a}$ & $63 a$ & $15,8 \mathrm{a}$ & $97 a$ & $14,9 \mathrm{a}$ \\
\hline 4 & $53,0 \mathrm{ab}$ & $23,0 \mathrm{bc}$ & $8,0 \mathrm{~b}$ & $5,0 \mathrm{a}$ & $\begin{array}{l}11,0 \\
a\end{array}$ & $63 a$ & $61 \mathrm{a}$ & $16,9 \mathrm{a}$ & $95 \mathrm{~b}$ & $14,2 \mathrm{~b}$ \\
\hline 5 & $33,0 \mathrm{~b}$ & $36,0 \mathrm{abc}$ & $26,0 \mathrm{ab}$ & $1,0 \mathrm{~b}$ & $4,0 \mathrm{a}$ & $26 \mathrm{c}$ & $18 \mathrm{c}$ & $3,3 \mathrm{c}$ & $62 d$ & $7,3 d$ \\
\hline 6 & $52,0 \mathrm{ab}$ & $6,0 \mathrm{c}$ & $34,0 \mathrm{a}$ & $1,0 \mathrm{~b}$ & $7,0 \mathrm{a}$ & $41 \mathrm{~b}$ & $38 \mathrm{~b}$ & $9,2 \mathrm{~b}$ & $84 \mathrm{c}$ & $10,3 \mathrm{c}$ \\
\hline CV\% & 19,8 & 26,8 & 30,4 & 57,7 & 42,6 & 16,8 & 18,4 & 19,6 & 29,8 & 18,8 \\
\hline
\end{tabular}

${ }^{1}$ Médias seguidas pela mesma letra na coluna não diferem entre si pelo teste de Tukey, a 5\%; CV=Coeficiente de variação.

Os resultados de emergência de plântulas e índice de velocidade de emergência classificaram os seis lotes em diferentes níveis de vigor, alternando a posição dos lotes 4 e 5 conforme o tamanho da semente, além disso, as médias obtidas foram superiores às do teste de germinação. Resultado semelhante foi obtido no trabalho de Menezes \& Silveira (1995), no qual acrescentaram que o teste de emergência quando realizado na época normal de semeadura para a cultura estudada, fornece as condições climáticas necessárias para a germinação das sementes. Segundo Rodo et al. (2001), este foi o único teste a detectar diferenças significativas entre os lotes analisados.

A diferença no teor de água inicial entre os lotes foi de $1 \%$ e após o procedimento tradicional (Tabela 3), essa variação foi de $6,2 \%$ excedendo o percentual limite de $4 \%$ indicado por Marcos Filho (1999) e contribuindo para o desenvolvimento de fungos em todos os lotes e períodos. Tal observação também foi relatada por Rodo et al. (2000). O uso da solução salina possibilitou que os níveis médios de variação ficassem em torno de $1,2 \%$, impedindo a proliferação de microrganismos e, ao mesmo tempo, propiciando que a temperatura fosse 0 fator relevante, em relação ao prolongamento do período de exposição durante 0 envelhecimento.

As médias verificadas nos testes de envelhecimento acelerado foram semelhantes, independentemente do tipo de solução, em função do tamanho das sementes (Tabela 4). Entretanto, no procedimento com água foi possível classificar os lotes em 3 classes enquanto que, no envelhecimento com solução salina obteve-se 4 diferentes níveis de vigor. Segundo Ribeiro (2000) o teste de envelhecimento com água demonstrou-se eficiente na avaliação de sementes de cenoura, alface e brócolos.

Em todas as avaliações, as sementes grandes apresentaram médias significativamente superiores às das pequenas. Vários autores têm relatado a superioridade do vigor das sementes maiores de cenoura, como Usik (1981) e Gray \& Steckel (1983). Por outro lado, Bittencourt (1991) e Rodo et al. (2001), não constataram diferenças de qualidade, em função do tamanho, em sementes de cenoura 'Brasília'. 
LIMA, C.B. \& ATHANÁZIO, J.C. Testes de vigor para sementes...

TABELA 3 - Teor de água (\%) de sementes de cenoura população 'Londrina', expostas aos testes de envelhecimento acelerado com água (EAA) e com solução salina (EAS).

\begin{tabular}{|c|c|c|c|c|c|c|c|}
\hline \multirow{2}{*}{ Testes } & \multirow{2}{*}{ Horas } & \multicolumn{6}{|c|}{ Lotes } \\
\hline & & 1 & 2 & 3 & 4 & 5 & 6 \\
\hline \multirow{8}{*}{ EAA } & Inicial & 9,5 & 8,7 & 8,9 & 9,7 & 8,9 & 8,9 \\
\hline & 12 & 46,9 & 26,5 & 25,4 & 25,9 & 30,6 & 26,2 \\
\hline & 24 & 47,1 & 52,3 & 39,9 & 39,1 & 45,5 & 38,2 \\
\hline & 36 & 38,4 & 38,5 & 57,1 & 44,0 & 42,2 & 38,0 \\
\hline & 48 & 40,0 & 39,3 & 41,6 & 39,7 & 37,6 & 41,2 \\
\hline & 60 & 58,8 & 58,3 & 56,0 & 54,0 & 52,2 & 54,2 \\
\hline & 72 & 47,7 & 42,3 & 44,8 & 47,0 & 44,6 & 42,7 \\
\hline & 84 & 56,6 & 59,0 & 52,0 & 50,0 & 48,3 & 47,7 \\
\hline \multirow[t]{4}{*}{ Variação média } & - & 38,5 & 36,4 & 36,3 & 33,1 & 34,1 & 32,3 \\
\hline & 12 & 12,2 & 11,7 & 10,8 & 11,1 & 10,8 & 11,8 \\
\hline & 24 & 12,1 & 12,3 & 13,4 & 10,1 & 12,2 & 11,5 \\
\hline & 36 & 12,4 & 10,9 & 11,1 & 11,4 & 10,6 & 13,0 \\
\hline \multirow[t]{4}{*}{ EAS } & 48 & 11,2 & 11,1 & 13,2 & 12,4 & 11,0 & 10,4 \\
\hline & 60 & 14,9 & 14,2 & 14,7 & 14,1 & 13,8 & 17,2 \\
\hline & 72 & 17,5 & 13,7 & 13,4 & 13,9 & 13,1 & 13,5 \\
\hline & 84 & 12,7 & 11,7 & 11,7 & 12,8 & 11,5 & 11,3 \\
\hline Variação média & - & 3,8 & 3,5 & 3,7 & 2,6 & 3,0 & 3,8 \\
\hline
\end{tabular}

TABELA 4 - Percentuais médios de germinação (G), primeira contagem da germinação (PCG) e velocidade de germinação (VG), obtidos após diferentes períodos de exposição aos testes de envelhecimento acelerado com água e com solução salina, de sementes de cenoura população 'Londrina'.

\begin{tabular}{|c|c|c|c|c|c|c|c|}
\hline & & \multicolumn{3}{|c|}{ Envelhecimento com água } & \multicolumn{3}{|c|}{ Solução Salina } \\
\hline & & $\mathrm{G}$ & PCG & VG & $G$ & PCG & VG \\
\hline \multirow{6}{*}{ Lotes } & 1 & $1 c^{1}$ & $0 \mathrm{c}$ & $0 \mathrm{c}$ & $0 \mathrm{~d}$ & $0 \mathrm{~d}$ & $0 \mathrm{~d}$ \\
\hline & 2 & $8 \mathrm{c}$ & $6 c$ & $2 c$ & $7 d$ & $4 d$ & $1 d$ \\
\hline & 3 & $62 \mathrm{a}$ & 59 a & $18 \mathrm{a}$ & $59 \mathrm{a}$ & $56 \mathrm{a}$ & $14 \mathrm{a}$ \\
\hline & 4 & $33 \mathrm{~b}$ & $31 \mathrm{~b}$ & $9 \mathrm{~b}$ & $36 \mathrm{~b}$ & $34 \mathrm{~b}$ & $9 \mathrm{~b}$ \\
\hline & 5 & $30 \mathrm{~b}$ & $26 \mathrm{~b}$ & $7 \mathrm{~b}$ & $27 c$ & $24 c$ & $6 c$ \\
\hline & 6 & $35 b$ & $31 \mathrm{~b}$ & $8 \mathrm{~b}$ & $28 c$ & $25 c$ & $6 c$ \\
\hline \multirow{8}{*}{ Horas } & 0 & $36 \mathrm{a}$ & $33 \mathrm{a}$ & $9 a$ & $36 \mathrm{a}$ & $33 \mathrm{a}$ & $9 \mathrm{a}$ \\
\hline & 12 & $30 a b$ & $27 a b$ & $7 \mathrm{ab}$ & $31 a b$ & $29 a b$ & $8 a b$ \\
\hline & 24 & $28 a b$ & $26 a b$ & $7 a b$ & $28 \mathrm{abc}$ & $26 a b c$ & $7 a b c$ \\
\hline & 36 & $31 a b$ & $29 a b$ & $9 \mathrm{a}$ & $28 \mathrm{abc}$ & $25 a b c$ & $6 \mathrm{bcd}$ \\
\hline & 48 & $23 b$ & $22 b$ & $6 a b$ & $20 c$ & $18 c$ & $4 d$ \\
\hline & 60 & $25 \mathrm{~b}$ & $23 \mathrm{~b}$ & $7 a b$ & $25 \mathrm{bc}$ & 22 bc & $5 \mathrm{~cd}$ \\
\hline & 72 & $22 \mathrm{~b}$ & $20 \mathrm{~b}$ & $5 b$ & $21 c$ & $19 c$ & $4 d$ \\
\hline & 84 & $29 a b$ & $27 a b$ & $8 a b$ & 22 bc & $20,0 \mathrm{bc}$ & $5 \mathrm{~cd}$ \\
\hline \multirow{2}{*}{ Tamanho } & Grandes & $33 a$ & $31 \mathrm{a}$ & $9 a$ & $30 a$ & $28 a$ & $7 a$ \\
\hline & Pequenas & $23 \mathrm{~b}$ & $20 \mathrm{~b}$ & $5 b$ & $22 \mathrm{~b}$ & $20 \mathrm{~b}$ & $5 b$ \\
\hline CV\% & & 37,6 & 41,7 & 43,7 & 41,1 & 44,3 & 44,0 \\
\hline
\end{tabular}

${ }^{\top}$ Médias seguidas pela mesma letra na coluna, não diferem entre si pelo teste de Tukey, a 5\%; CV= Coeficiente de variação.

Scientia Agraria, Curitiba, v.10, n.6, p.455-461, Nov./Dec. 2009. 
LIMA, C.B. \& ATHANÁZIO, J.C. Testes de vigor para sementes...

Existe divergência em relação ao tempo de exposição das sementes de cenoura às condições do teste de envelhecimento acelerado. Rodo et al. (2000) indicaram o período de $72 \mathrm{~h}$ e o uso de $\mathrm{NaCl}$, entretanto, Lima et al. (2004) concluíram que esse período foi excessivo. No presente estudo, pode-se verificar uma semelhança entre os resultados observados nos períodos de 48 e $72 \mathrm{~h}$, porém, o uso da solução salina, possibilitou uma melhor classificação dos lotes em função dos diferentes níveis de vigor.
Não apresentaram correlação significativa tanto para sementes grandes, quanto para pequenas, os testes de emergência de plântulas com os índices de velocidade de emergência e de germinação. Entre as sementes pequenas, o teste de tetrazólio não mostrou correlação significativa com os índices de velocidade de germinação e de emergência. Os demais testes apresentaram altos coeficientes de correlação, indicando que podem ser utilizados para avaliação do vigor de sementes de cenoura (Tabela 5).

TABELA 5 - Coeficientes de correlação simples entre os dados obtidos através de diferentes testes, na avaliação da qualidade fisiológica de sementes de cenoura população 'Londrina', separadas por tamanho.

\begin{tabular}{|c|c|c|c|c|c|c|c|c|}
\hline Tamanho & Testes & PCG & IVG & EP & IVE & $\mathrm{TZ}$ & EAA & EAS \\
\hline \multirow{8}{*}{ Grandes } & $\mathrm{G}$ & $0,99^{* *}$ & $0,99^{\star *}$ & $0,97^{\star \star}$ & $0,98^{* \star}$ & $0,92^{* \star}$ & $0,95^{* *}$ & $0,92^{* \star}$ \\
\hline & EAS & $0,94^{* *}$ & $0,74^{* *}$ & $0,75^{\star *}$ & $0,70^{*}$ & $0,88^{* *}$ & $0,92^{* *}$ & - \\
\hline & EAA & $0,96^{* *}$ & $0,74^{\star *}$ & $0,78^{* *}$ & $0,70^{*}$ & $0,91^{* *}$ & - & \\
\hline & $\mathrm{TZ}$ & $0,96^{* *}$ & $0,70^{\star}$ & $0,86^{* *}$ & $0,70^{*}$ & - & & \\
\hline & IVE & $0,70^{*}$ & $0,97^{* *}$ & $0,53^{\mathrm{ns}}$ & - & & & \\
\hline & EP & $0,87^{* *}$ & $0,55^{\mathrm{ns}}$ & - & & & & \\
\hline & IVG & $0,72^{* *}$ & - & & & & & \\
\hline & PCG & - & & & & & & \\
\hline \multirow{8}{*}{ Pequenas } & $\mathrm{G}$ & $0,99^{\star \star}$ & $0,98^{\star \star}$ & $0,95^{\star \star}$ & $0,99^{\star \star}$ & $0,93^{\star *}$ & $0,89^{*}$ & $0,93^{\star *}$ \\
\hline & EAS & $0,96^{* *}$ & $0,72^{* *}$ & $0,77^{\star *}$ & $0,70^{*}$ & $0,83^{* *}$ & $0,91^{* *}$ & - \\
\hline & EAA & $0,95^{\text {** }}$ & $0,70^{*}$ & $0,78^{* *}$ & $0,70^{*}$ & $0,83^{* *}$ & - & \\
\hline & $\mathrm{TZ}$ & $0,91^{* *}$ & $0,50^{\mathrm{ns}}$ & $0,85^{\star *}$ & $0,48^{\text {ns }}$ & - & & \\
\hline & IVE & $0,71^{*}$ & $0,98^{* *}$ & $0,53^{\text {ns }}$ & - & & & \\
\hline & EP & $0,85^{\text {** }}$ & $0,54^{\mathrm{ns}}$ & - & & & & \\
\hline & IVG & $0,72^{* *}$ & - & & & & & \\
\hline & PCG & - & & & & & & \\
\hline
\end{tabular}

**significativo a 1\%; * significativo a 5\%; ns=não significativo; G=germinação; PCG=primeira contagem da germinação;

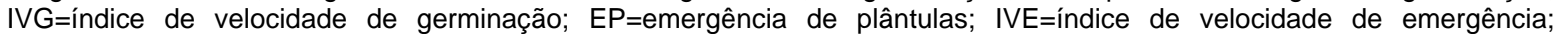
TZ=tetrazólio; EAA=envelhecimento acelerado com água; EAS=envelhecimento acelerado com solução salina.

O índice de velocidade de emergência e a emergência de plântulas, não apresentaram coeficientes de correlação significativos, apesar de possibilitarem o mesmo nível de distinção entre os lotes. Segundo Lima (1993), o teste de correlação não deve ser usado isoladamente para estimar a eficiência dos testes de vigor, podendo promover a obtenção de informações incompletas ou incorretas, pois, a correlação significativa indica uma tendência de variação semelhante entre dois testes, não significando, porém, que há uma correspondente precisão de estimativa da qualidade fisiológica do lote.

\section{CONCLUSÕES}

O teste de emergência de plântulas destaca-se na classificação dos lotes quanto às diferenças de vigor. A combinação solução salina com 48 ou 72 h de exposição é a mais adequada para o teste de envelhecimento acelerado. As sementes de maior tamanho apresentam qualidade fisiológica superior às menores.

\section{REFERÊNCIAS}

1. ANDRADE, R. N. B. et al. Testes de germinação e de tetrazólio em sementes de cenoura armazenadas por diferentes períodos. Revista Brasileira de Sementes, v. 18, n. 1, p. 108-116, 1996.

2. BITTENCOURT, M. L. C. Qualidade das sementes e avaliação das progênies de meios-irmãos de cenoura (Daucus carota L.) 'Brasília'. 1991. 77 f. Dissertação. (Mestrado em Ciências Agrárias) - Universidade Federal de Viçosa, Viçosa, 1991.

3. BRASIL. Ministério da Agricultura e Reforma Agrária. Regras para análise de sementes. Brasília: SNDA/DND/CLAV, 1992. $365 \mathrm{p}$.

4. FRANZIN, S. M. et al. Métodos para avaliação do potencial fisiológico de sementes de alface. Revista Brasileira de Sementes, v. 26, n. 2, p. 63-69, 2004 
5. GRAY. D.; STECKEL, J. R. A. Some effects of umbel order and harvest date on carrot seed variability and seedling performance. Journal Horticutural Science, v. 58, n. 1, p. 73-82, 1983

6. HILHORST, H. W. M. et al. Curso avançado em fisiologia e tecnologia de sementes. Lavras: UFLA, 2001. 74 p.

7. JIANHUA, Z.; McDONALD, M. B. The salturated salt accelerated aging test for small-seeded crops. Seed Science and Technology, v. 25, n. 1, p. 123-131, 1996.

8. LIMA, C. B. et al. Vigor e germinação de sementes de cenoura e cebola submetidas a diferentes números de horas de envelhecimento acelerado. In: CONGRESSO BRASILEIRO DE OLERICULTURA, 44., 2004, Campo Grande. Resumos... Campo Grande: Horticultura Brasileira, 2004. 1 CD-ROM.

9. LIMA, D. Avaliação da viabilidade e vigor de sementes de cebola (Allium cepa L.). 1993. 61 f. Dissertação. (Mestrado em Agronomia) - Universidade Federal de Pelotas, Pelotas, 1993.

10. MARCOS FILHO, J. Teste de envelhecimento acelerado. In: KRZYZANOWSKI, F.C.; VIEIRA, R.D.; FRANÇA NETO, J.B. (Ed.). Vigor de sementes: conceitos e testes. Londrina: ABRATES, 1999. p.1-24.

11. MENEZES, N. L.; SILVEIRA, T. L. D. Métodos para avaliar a qualidade fisiológica de sementes de arroz. Scientia Agricola, v. 52, n. 2, p. 350-359, 1995.

12. MUNIZ, M. F. B. et al. Qualidade fisiológica e sanitária de sementes de melão (Cucumis melo). Ciência Rural, v. 34, n. 3 , p. 951-953, 2004.

13. POPINIGIS, F. Fisiologia de sementes. 2.ed. Brasília:AGIPLAN,1985. 289 p.

14. RAMOS, N. P. et al. Envelhecimento acelerado em sementes de rúcula (Eruca sativa L.). Revista Brasileira de Sementes, v. 26, n. 1, p. 98-103, 2004.

15. RIBEIRO, F. C. Comparação entre os sistemas de envelhecimento artificial de sementes através de soluções salinas e o tradicional em cenoura (Daucus carota L.), alface (Lactuca sativa L.) e brócolos (Brassica oleracea variedade itálica Plenk). 2000. 56 f. Dissertação (Mestrado em Agronomia) - Faculdade de Ciências Agrárias e Veterinárias, Universidade Estadual Paulista, Jaboticabal, 2000.

16. RODO, A. B. et al. Qualidade fisiológica e tamanho de sementes de cenoura. Scientia Agricola, v. 58, n. 1, p. 201-204, 2001

17. RODO, A. B.; PANOBIANCO, M.; MARCOS FILHO, J. Metodologia alternativa do teste de envelhecimento acelerado para sementes de cenoura. Scientia Agricola, v. 57, n. 2, p. 289-292, 2000.

18. USIK, G. E. Effect of seed size on emergence and yield in onions and carrots. Horticultural Abstracts, v. 51, n. 3, p.155156, 1981.

19. VIEIRA, J. V. et al. Seleção de progênies de meio-irmãos de cenoura baseada em características de sementes. Horticultura Brasileira, v. 23, n. 1, p. 44-47, 2005.

Recebido em 13/02/2009

Aceito em 14/08/2009 
